\title{
Comparative genomic analyses provide clues to capsule switch in Streptococcus suis
}

4 Yinchu Zhu ${ }^{1,2,3,4, *, \#}$, Wenyang Dong ${ }^{2,3,4,5, *}$, , Jiale Ma ${ }^{2,3,4}$, Yue Zhang ${ }^{2,3,4,6}$, Xiaojun Zhong ${ }^{2,3,4,7}$,

5 Zihao Pan ${ }^{2,3,4}$, Guangjin Liu ${ }^{2,3,4}$, Zongfu $\mathrm{Wu}^{2,3,4}$, Huochun $\mathrm{Yao}^{2,3,4}$, *

6

$7{ }^{1}$ Institute of Animal Husbandry and Veterinary Sciences, Zhejiang Academy of Agricultural

8 Sciences, Hangzhou 310021, China

$9{ }^{2}$ College of Veterinary Medicine, Nanjing Agricultural University, Nanjing 210095, China

$10{ }^{3}$ Key Lab of Animal Bacteriology, Ministry of Agriculture, Nanjing 210095, China

$11 \quad{ }^{4}$ OIE Reference Lab for Swine Streptococcosis, Nanjing 210095, China

$12{ }^{5}$ Beijing Advanced Innovation Center for Genomics (ICG) \& Biomedical Pioneering Innovation

13 Center (BIOPIC), Peking University, Beijing 100871, China

$14{ }^{6}$ College of Animal Science and Veterinary Medicine, Henan Agricultural University,

15 Zhengzhou 450002, China

$16{ }^{7}$ College of Animal Science and Technology, College of Veterinary Medicine, Zhejiang A \& F

17 University, Hangzhou, 311300, China

$18{ }^{\#}$ Authors contributed equally to this study

19 *Correspondence: zhuyinchu@ zaas.ac.cn (Y. Z.), dongwenyang@ pku.edu.cn (W. D.),

20 yaohch@njau.edu.cn (H. Y.) 


\section{Abstract}

22 Streptococcus suis (S. suis) is a major bacterial pathogen in swine industry and also an emerging

23 zoonotic agent. S. suis produces an important extracellular component, capsular polysaccharides

24 (CPS). Based on which, dozens of serotypes have been identified. Through virulence genotyping,

25 we uncovered the relatedness between proportions of SS2, SS3 and SS7 strains despite their

26 differences in serotypes. Multi-locus sequence typing (MLST) was used to characterize whole $S$.

27 suis population, revealing that there is capsule switch between S. suis strains. Importantly,

28 capsule switch occurred in SS2, 3 and 7 strains belonging to CC28 and CC29, which is

29 phylogenetically distinct from the main CC1 SS2 lineage. To further explore capsule switch in $S$.

30 suis, comparative genomic analyses were performed using available $S$. suis complete genomes.

31 Phylogenetic analyses suggested that SS2 strains can be divided into two clades (1 and 2), and

32 those classified into clade 2 are colocalized with SS3 and SS7 strains, which is in accordance

33 with above virulence genotyping and MLST analyses. Clade 2 SS2 strains presented high genetic

34 similarity with SS3 and SS7 and shared common competence and defensive elements, but are

35 significantly different from Clade 1 SS2 strains. Notably, although the cps locus shared by Clade

361 and 2 SS2 strains is almost the same, a specific region in cps locus of strain NSUI002 (Clade 2

37 SS2) can be found in SS3 cps locus, but not in Clade 1 SS2 strain. These data indicated that SS2

38 strains appeared in CC28 and CC29 might acquire cps locus through capsule switch, which could well explain the distinction of genetic lineages within SS2 population.

Key words: Streptococcus suis; Capsule switch; S. suis serotype 2; S. suis serotype 3; S. suis 


\section{Introduction}

Streptococcus suis (S. suis) is a major bacterial pathogen causing global economic losses to swine industry. It is also a serious zoonotic pathogen in countries with intensive swine production. Capsule polysaccharide (CPS) is the key virulence determinant in S. suis, which contributes to the bacterial resistance to host immunity (Fittipaldi et al., 2012). Sequence analyses of $S$. suis genomes revealed a cps locus with variable lengths and a series of genes specific to CPS production (Okura et al., 2013). It is thought that CPS of S. suis is synthesized and exported through the Wzx/Wzy pathway, which is a mechanism commonly used in

Streptococcus pneumoniae

(S. pneumoniae) and Streptococcus agalactiae

(S. agalactiae)

\section{3} capsular biosynthesis (Yother, 2011). The difference in cps locus would lead to the difference in components and structures of CPS, and importantly, the serum antigenicity. In the 1980s and 1990s, 35 serotypes (types 1 to 34 and type 1/2) having been described based on CPS antisera coagglutination test (Higgins et al., 1995). Later on, serotypes 20, 22, 26, 32, 33 and 34 are suggested to be removed from S. suis species (Hill et al., 2005; Tien et al., 2013). More recently, a novel variant serotype $\mathrm{Chz}$ and other 8 novel cps loci harboring specific wzy polymerase genes and $w z x$ flippase genes were identified (Pan et al., 2015; Qiu et al., 2016), revealing the high diversity of cps locus in S. suis genomes.

Among the known serotypes worldwide, S. suis serotypes 2, 3, 9, 7, 8, 4 and 1 are the most prevalent serotypes linked with infection of swine, especially serotype 2 (SS2) (GoyetteDesjardins et al., 2014). In North America, serotype 2 and 3 are the two most prevalent serotypes isolated from clinical pig cases (Goyette-Desjardins et al., 2014). In Asia, the most prevalent serotypes in infected pigs are serotypes 2, 3, 4, 7 and 8 (Goyette-Desjardins et al., 2014; Wei et al., 2009). In Europe, serotype 9 and 2 are more frequently found in clinical pig cases, followed 
67 by serotypes 7, 8, 3 and 1 (Goyette-Desjardins et al., 2014). As for the infection of human,

68 serotypes $2,4,5,9,14,16,21$ and 24 have already been reported, of which SS2 is the

69 predominate serotype (Goyette-Desjardins et al., 2014; Kerdsin et al., 2017). Therefore, most

70 studies in S. suis field are focusing on SS2 due to its close link with diseases. However,

71 heterogenicity in SS2 population has been observed, indicating serotyping alone is not sufficient

72 to characterize $S$. suis strains.

73 In addition to capsule serotyping, multilocus sequence typing (MLST) is most the widely used

74 typing method in epidemiological studies of S. suis. Analyses of MLST data from different

75 sources determined the sequence types (STs) of stains, which could be further clustered into

76 clonal complexes (CCs) (King et al., 2002). Interestingly, MLST analyses of S. suis isolates

77 suggested that SS2 population can be divided into two major lineages, not only in terms of

78 geographical and genetic background, but also virulence phenotype (Fittipaldi et al., 2011;

79 Goyette-Desjardins et al., 2014; Yao et al., 2015; Zhu et al., 2013). Among SS2 isolates,

80 although ST1/ST7 (CC1), ST25 (CC29) and ST28 (CC28) strains have been isolated in both

81 Asia and North American, CC1 strains are more prevalent in Asia, while CC28 and CC29 strains

82 are more commonly found in North America (Goyette-Desjardins et al., 2014). Importantly,

83 ST1/ST7 strains are significantly more virulent than ST25 and ST28 strains (Athey et al., 2015;

84 Fittipaldi et al., 2011; Guo et al., 2020). Although the distinctions of genetic and virulence

85 phenotypic lineages within SS2 population have been found, the way it was formed are not yet

86 fully understood.

87 Capsule switch, a change of serotype of a single clone by alteration or exchange of its cps locus,

88 has been identified in streptococcal species including Streptococcus iniae (S. iniae) (Heath et al.,

89 2016), S. pneumoniae (Wyres et al., 2013) and S. agalactiae (Martins et al., 2010). Development 
of MLST greatly promote the studies on capsular switch, which can be more easily identified by

91 detecting strains of different serotypes sharing the same ST. Although capsular switch has been

92 found in S. suis isolates (King et al., 2002), the effects of this phenomena on S. suis population

93 structure has not been demonstrated. Previously, we showed that the virulence related genes are

94 differently distributed in strains of two SS2 clusters (Dong et al., 2015), which is in accordance

95 with the known SS2 sequence type classification (Fittipaldi et al., 2011; Zhu et al., 2013). In this

96 study, we combined MLST analysis, virulence genotyping and whole genome analysis, to

97 explore the capsular switch in S. suis, and highlight its potential role of in shaping SS2

98 population.

\section{Materials and methods}

100

\subsection{Bacteria strains and culture conditions}

101 All S. suis serotype 3 (SS3) and S. suis serotype 7 (SS7) strains are the field strains isolated from

102 China from 2004 to 2018 and were stored in our laboratory. The typical virulent SS2 strain

103 ZY0719 was isolated from a diseased pig during an outbreak in China. S. suis was grown in

104 Todd-Hewitt broth medium (THB; Becton Dickinson, Sparks, MD, USA) at $37^{\circ} \mathrm{C}$ overnight.

105 The antibiotics including spectinomycin $(100 \square \mu \mathrm{g} / \mathrm{ml})$ and chloramphenicol $(5 \mu \mathrm{g} / \mathrm{ml})$ were

106 added into the medium if it is needed. The plasmids for Streptococcus pSET-2::cat was used in

107 this experiment for DNA template. A detailed information for bacterial strains used in this study

108 listed in Table S1.

\subsection{PCR assays}

110 A previously established species-specific polymerase chain reaction (PCR) based on the gdh and

111 recN genes were performed to confirm the identification of S. suis (Okwumabua et al., 2003).

112 The serotype-specific PCR was used to identify SS3 and SS7 strains among the collected S. suis 
113 isolates (Kerdsin et al., 2014). In the virulence genotyping assay, 19 S. suis virulence-associated

114 genes, including mrp, epf, sly, rgg, ofs, srtA, pgdA, gapdh, iga, endoD, ciaRH, salKR, manN,

115 purD, dppIV, neuB, dltA, comR and $s c n F$ were detected by individual PCR as previously

116 described (Dong et al., 2015; Dong et al., 2017). In MLST assays, seven housekeeping genes

117 aroA, mutS, cpn60, dpr, recA, thrA and gki were amplified by PCR as described previously

118 (King et al., 2002), and the amplification fragments were sequenced.

\subsection{Clustering analysis}

For MLST, allele numbers and sequence types (STs) were identified in MLST database

121 (http://ssuis.mlst.net/). The eBURST (http://eburst.mlst.net) program was used to determine

122 population structures through identifying potential clonal complexes (CCs) and founders. For

123 virulence genotyping, BioNumerics (version 6.6, Applied Maths, Kortrijk, Belgium) was used to

124 analyze the profiles of virulence related genes as previously described (Dong et al., 2015; Dong

125 et al., 2017; Mateus et al., 2013; Zhu et al., 2017): the resemblance was computed with simple

126 matching coefficients, and agglomerative clustering was performed using the unweighted

127 average linkage (UPGMA). The profiling of SS2 virulence related genes used in this clustering

128 analysis is acquired from a previous study (Dong et al., 2015).

\subsection{Phylogenetic analysis}

130 The complete genome sequences of 25 S. suis isolates of different serotypes were obtained from

131 NCBI GenBank and used for phylogenetic analyses, including the 3 S. suis genomes provided by

132 our research group (ZY05719, T15 and SC070731). A phylogenetic tree based on the 1373

133 single copy orthology clusters generated from clustering of the 25 strains was constructed using

134 neighbor-Joining (NJ) method. The information of strains were listed in table S2. 


\subsection{Multi-genome alignment analysis}

136 Comparative analysis of alignments among S. suis genomes were conducted using the

137 progressive alignment option of the Mauve software (Darling et al., 2004). ZY05719 was 138 selected as virulent SS2 strains, NSUI002 and NSUI060 were the typical avirulent SS2 strains.

139 Genomes of SS3 strains YB51 and ST3, SS7 strain D9 were also used in genome alignments.

\subsection{Average nucleotide identity analysis}

141 The genomic similarity between SS2, SS3 and SS7 strains was evaluated by average nucleotide

142 identity (ANI) method. The algorithm implemented at the EzGenome server was used To

143 calculate the ANI value (www.ezbiocloud.net/tools/ani). The proposed and generally accepted 144 species boundary for ANI value are 95 96\% (Richter and Rossello-Mora, 2009).

\subsection{Defense elements analysis}

146 Bacterial defense systems were ancient elements that confers resistance to foreign genetic 147 elements, including Restriction-Modification (RM) system and Clustered Regularly Interspaced 148 Short Palindromic Repeats (CRISPR) system. RM elements and CRISPR components in S. suis 149 were determined through CRISPRs finder (http://crispr.u-psud.fr/) and web-service REBASE 150 (http://rebase.neb.com/rebase/rebase.html), representatively. The structures of RM and CRISPR 151 systems are achieved through performing an all-to-all BLASTN search in the NCBI nucleotide 152 database. Consensus sequences of repeat sequences in defense systems were determined using 153 MegAlign.

\section{$154 \quad 2.8$ Competence system analysis}

155 S. suis is harbors a natural competence system, which greatly facilitates the DNA exchange 156 through horizontal genetic transfer. In our previous study, we identified that ComRS-ComX 157 competence system varies in different genotype background S. suis strains (Zhu et al., 2019). 

genomes. The amino acid sequence of competence systems was visualized by DNAMAN software. To experimentally validate the functional difference between $S$. suis strains, a transformation efficiency test was performed between 8 selected SS2, SS3 and SS7 field strains stored in our laboratory. Briefly, two different synthetic competence peptides XIP (type A:GNWGTWVEE and type B: LGDENWWVK) were added to the bacterial culture with template DNA (plasmid pSET-2::cat) to induce the natural transformation. Competency was calculated based on the amount of transformants that grew in THB plates with spectinomycin and chloramphenicol selection.

\subsection{Analysis of cps locus}

The diversity serotypes are based on the variation of genetic locus harboring capsular

polysaccharide related genes (cps locus). In general, strains with a same serotype would present highly similar genomic sequence in cps locus. The sequence of cps locus in genomes of CC1 SS2 strain ZY05719, CC28 SS2 strain NSUI002, and CC28 SS3 strain ST3 were determined using BLAST. Corresponding sequences and gene annotation information were obtained from obtained from NCBI GenBank. The homologous analysis was performed using Easyfig software.

174 3. Results

$175 \quad 3.1$ Virulence genotyping of field strains revealed relatedness of a SS2 subpopulation with SS3 and SS7

177 Virulence genotyping is a powerful tool to study pathogenic bacteria, which can contribute to 178 screen for specific disease-associated virulence genes (Gerjets et al., 2011; Rasmussen et al., 179 2013) or uncover the relatedness between isolates (Dong et al., 2017; Mateus et al., 2013). 
181

182

183

184

185

186

187

188

189

190

191

192

193

194

195

196

197

198

199

200

201

202

203

genes in SS2 isolates, and revealed that SS2 strains can be divided into two clusters due to the different distribution of genes epf, sly, endoD, rgg and scnF (Dong et al., 2015). In this study, we further detected the presence of virulence related genes in SS3 and SS7 isolates by PCR, and performed clustering analysis based on the gene profiles of SS2 (n=62), SS3 (n=17) and SS7 $(n=9)$ isolates (Figure 1). In accordance with what we have showed previously (Dong et al., 2015), SS2 strains could be divided into two clusters ((I and II) with a different prevalence of virulence related genes. Interestingly, SS3 and SS7 isolates were classified into cluster II together with one SS2 subpopulation, suggesting a relatedness of SS3 and SS7 with that SS2 subpopulation (Figure 1). For the gene distribution, 8 genes were detected in all $S$. suis field isolates, namely $s r t A, p g d A$, dltA, iga, sspA, manN, ciaHR and gapdh; whereas gene epf and $r g g$ were only detected in cluster I but not in cluster II. According to virulence genotyping result in this study and previous knowledge (Dong et al., 2015; Dong et al., 2017; Kobayashi et al., 2013; Mateus et al., 2013), we hypothesized that the presence of similar virulence genotypes reflects phylogenetic relatedness of SS2, SS3 and SS7. Therefore, we performed genetic analyses in $S$. suis population to further test our hypothesis.

\subsection{MLST analysis demonstrated capsule switch in S. suis between SS2, SS3 and SS7}

MLST is an important and widely used molecular method in studying S. suis epidemiology, in which seven housekeeping genes are sequenced to assess the genomic variation and define Sequence Types (STs). The availability of updated information in S. suis MLST database (http://ssuis.mlst.net/) makes it possible to apply a grouping approach for identification of Clonal Complex (CC) and perform comparative analysis with strains of different serotypes. A total of 1528 S. suis strains (701 STs) from MLST database was diagramed by eBURST on the basis of their allelic profiles. The eBURST analysis revealed 9 major clonal complexes (Figure 2A). CC1 
$204(629 / 1528)$ is the predominant clonal complexes in S. suis, followed by CC16 (120/1528), CC28

205 (71/1528), CC29 (62/1528) and CC104 (30/1528), whereas CC94, CC528, CC423 and CC201

206 are much smaller schemes.

207 The MLST analysis revealed capsular switch in S. suis, that strains of different serotypes sharing

208 the same ST. In S. suis, capsular switch was firstly reported in 2002 (King et al., 2002), which

209 analyzed the capsular switch events from ST1 to ST92, and showed it occurred in ST1, 13, 16,

$21017,27,28,29,65$ and 76. Our updated analysis identified novel STs with capsular switch,

211 including ST15, 89, 94, 105, 136, 156, 243 and 297 (Table 1). Importantly, there is capsular

212 switch within SS2, SS3 and SS7 strains. ST27 from CC28 has both SS2 and SS3 strains, and

213 ST29 from CC29 has SS2, SS3 and SS7 strains (Table 1 and Figure 2B). CC28 and CC29 have

214 already been demonstrated to be important SS2 lineages (Athey et al., 2015; Fittipaldi et al.,

215 2011). Interestingly, as shown in our eBURST analysis, CC28 harbors a SS3 major population,

216 and CC29 harbors a SS7 major population (Figure 2B). Those results suggested that a sub-

217 population of SS2 strains of CC28 and CC29 are more closely related to SS3 and SS7 strains,

218 and there is capsular switch in those two clonal complexes.

3.3 Whole genome phylogenetic analysis of different serotype strains deciphered two

\section{distinct clades in SS2}

221 To investigate the phylogenetic relationships among S.suis strains, we used 25 complete

222 genomes of different serotypes from GenBank dataset. The phylogenetic tree, constructed using

223 neighbor-joining (NJ) method (Figure 3), showed that S.suis strains of different serotypes can be

224 classified into three major clades (Clade 1, 2 and 3). SS1 strain, SS4 strain and SS2 avirulent

225 strain T15 appeared to be phylogenetically independent, whereas SS16 strain and SS9 strains are

226 located together in Clade 3. 
SS14 strain JS14 and SS1/2 strain SS12 are grouped together with ten SS2 strains, and formed

228

229

230

231

232

233

234

235

236

237

238

239

240

241

242

243

244

245

246

247

248

249

the largest clade (Clade 1) on phylogenetic tree. SS2 strains in this clade presented a short evolutionary distance from each other, suggesting that these strains were probably derived from a recent common ancestor. This result is in accordance with the MLST analysis, that all SS2 strains in Clade 1 belong to CC1. SS14 strain JS14 and SS1/2 strain SS12 also presented CC1 related STs, indicating a close link with Clade 1 SS2 strains.

Clade 2 included SS7 strain D9, SS3 strains ST3 and YB51, and four SS2 strains. These SS2 strains therefore showed a large divergence from Clade1 SS2. SS2 strain NSUI060 is a strain of ST25 (CC29), and is assigned in a same branch with ST29 (CC29) strain D9 on phylogenetic tree. SS2 strains 90-1330, 05HAS68 and NSUI002 belongs to ST28 (CC28), and are grouped in a same branch with two ST 35 (CC28) SS3 strains. Above result indicated that CC28/29 SS2 strains in this clade were more closely related with SS7 strain and SS3 strains rather than CC1 SS2 strains in Clade 1. This is consistent with the virulence genotyping result, and also reflecting the capsule switch identified by MLST within SS2, SS3 and SS7strains. Therefore, we further performed whole-genome comparative analysis to decipher capsule switch in S. suis.

\subsection{Clade 2 SS2 had a higher genomic similarity with SS3 and SS7 than Clade 1 SS2}

The arrangement and collinearity of Clade 1 and Clade $2 S$. suis genomes were investigated using Mauve program. We first used CC28 SS2 strain NSUI002 as reference genome, to compare clade 1 CC1 SS2 strain ZY05719, as well as CC28 SS3 strains ST3 and YB51 that colocalized with NSUI002 in Clade 2 sub-branch. Mauve analysis (Figure 4) of those strains indicated that rearrangements occurred in genomes of those strains, but the overall genomic organizations are relatively comparable. The NSUI002 and YB51 genomes showed more collinear than ZY05719, suggesting that NSUI002 and SS3 strains have a higher genomic 
250 similarity. We further compared the genomic organization of SS2 strain NSUI060 and SS7 strain

251 D9 (Figure S1), which are colocalized in a subgroup of Clade 2 as well. Alignment of the

252 genome sequences of NSUI060 and D9 revealed a high level of genomic rearrangement,

253 including large-scale deletion, insertion, translocation, and inversion. However, the genomes of

254 NSUI060 and D9 still shared a more similar sequence synteny with each other, but significantly

255 different from ZY05719 with respect to both collinearity and genome structure. Those results

256 show that Clade 2 SS2 strains and SS3/7 strains has the smaller scale of arrangements and higher

257 level of synteny compared with Clade 1 SS2.

258 The genomic relationship between Clade 1 isolates and Clade 2 isolates was further evaluated by 259 nucleotide sequence similarity. The average nucleotide identity (ANI) were calculated (Figure 5) 260 using the web-based EZ BioCloud platform ( www.ezbiocloud.net/tools/ani). The ANI values 261 between the Clade $1 \mathrm{SS} 2$ isolates are very high, ranged from 99.54 to $99.97 \square \%$, whereas the

262 ANI values between Clade $1 \mathrm{SS} 2$ isolates and Clade 2 SS2 is only from 96.28 to 96.94, which is 263 close to the cut-off values recommended for species delineation (95-96卬\%) (Richter and 264 Rossello-Mora, 2009). The Clade 2 SS2, SS3 and SS7 strains have ANI values above 98, and can 265 be further divided into two sub-clusters, which is in accordance with the phylogenetic analysis 266 and Mauve result. Especially, the ANI values between three Clade 2 SS2 isolates, namely 90267 1330, 05HAS68 and NSUI002, and SS3 strains are from 99.57 to 99.70, while the ANI value 268 between SS2 strain NSUI060 and SS7 strain D9 is 99.50. Thus, these results revealed the 269 genomic dissimilarity of Clade 1 SS2 isolates and Clade 2 SS2 isolates, supporting the 270 conclusion that Clade 2 SS2 isolates have a high relatedness with SS3 and SS7 strains, as 271 implied by the above whole genome comparison results. 
273 The defense elements, including RM system and CRISPR/Cas system, were detected in S. suis

274 genomes. Both of the systems have a role in protecting bacteria against invading exogenous

275 DNA, but the mechanism is different (Dupuis et al., 2013). The type I RM system uses HsdS, a

276 single protein can respond to the methylation of target sequence, to determine the specificity of

277 both restriction and methylation by the action of endonuclease HsdR and methyltransferase

278 HsdM (Willemse and Schultsz, 2016). In S. suis, a total of three type I RM systems are detected

279 in S. suis (Figure 6A). The Type A and Type B RM systems specifically appear in the Clade 1

280 SS2 strain, but not in Clade 2 strains. Type C RM system is present and conserved in strains of

281 both clades. However, Clade 2 strains have an inserted fic gene between the $h s d$ genes, which is

282 different from Clade 1 strains. Another defense element, CRISPR/cas system, provides acquired

283 immunity in prokaryotic organisms. It integrates short sequences of invading exogenous DNA

284 between CRISPR repeats, and cleaves reinvading foreign DNA when recognize same sequences

285 (Wiedenheft et al., 2012). CRISPR analysis result showed that the CRISPR components are

286 absent in Clade 1 strains but present in all Clade2 strains. The CRISPR repeat in Clade 2 strains

287 is 36 bp in length (GTTTTACTGTTACTTAAATCTTGAGAGTACAAA AAC), but SS7 strain

288 D9 has an additional variant form with an additional TTA at the end of the repeat (Figure 6B and

289 C). Above data is in accordance with a previous report (Okura et al., 2017), that SS3 and SS7

290 strains shared defense elements with Clade 2 SS2 strains, which is different from Clade 1 SS2

291 strains.

$292 \quad 3.6$ SS3, SS7 and Clade 2 SS2 shared a competence system different from Clade 1 SS2

293 S. suis is a bacterium with natural transformation ability, which depends on the ComRS

294 competence systems. Natural transformation contributes to the horizontal gene transfer in S. suis

295 and increase the diversity of $S$. suis genomes, which confers a unique advantage for capsule 
switch. Previously, we have identified three types of ComRS systems with specific competence pheromone in S. suis (Zhu et al., 2019). BLAST search results suggested that all Clade 1 isolates harbors Type A ComRS system, while all Clade 2 strains harbors Type B ComRS system (Figure 6C). We further detected the distribution of ComRS systems in field strains used in virulence genotyping (Figure 1). Results showed that all field strains that classified into cluster I in virulence genotyping have Type A ComRS system, whereas all strains classified into cluster II, including SS3 and SS7 isolates, harbor Type B ComRS system (data not shown). To experimentally test the transformation efficiencies of strains stimulated with noncognate synthetic competence pheromones, we randomly selected two cluster II SS2 strains (ZJJX0908005 and ZJ92091101), two cluster II SS3 strains (128-1-2 and 129-1-3), two cluster II

SS7 strains (SH59 and SH04815) and two representative cluster I SS2 strains (ZY05719 and SS3 and SS7, but could not respond to the pheromone from cluster I SS2 strains, which is consistent with phylogenetic analyses results.

\subsection{Analysis of cps locus provided evidence for capsule switch between SS3 and Clade 2 SS2}

313 To precisely characterize the capsular switch between SS2, SS3 and SS7, we compared the cps

314 genes locus of the representative S.suis strains. Although SS7 strain D9 is closely related to ST25

315 SS2 strain NSUI060, BLAST search on the D9 genome showed that the cps locus is disrupted

316 due to genomic rearrangement, and the cps genes are separated and translocated to different 317 genomic segments (data not shown). Therefore, here we only compared representative SS2 and 
319 (Figure 8). Sequence alignment of cps locus and its flanking regions of these strains revealed that

320 the $7.2 \mathrm{~kb}$-long upstream flanking sequence (tetR to $y a a A$ and $\operatorname{cps} A B C D$ ) and the $12.6 \mathrm{~kb}-\mathrm{long}$

321 downstream flanking sequence (aroA to $a s n S)$ are extremely conserved among S. suis isolates.

322 This suggested that the potential capsule switch event may occur through homologous

323 recombination in these regions probably independently of the overall genetic background. Except

324 for a translocated insertion of transposon element (red arrow), the SS2 cps locus of NSUI002 is

325 almost identical to that of ZY05719, and differs from SS3 cps locus. However, in the ending

326 region of SS2 cps locus, genes cps $2 T$, cps $2 U$ and $\operatorname{cps} 2 V$ are absent from the cps locus of

327 NSUI002. Instead, NSUI002 has a fragment shared high identity with sequence from SS3 cps

328 locus containing cps3O, cps3P and tnp3-4 (red line box). It is worth noting this unique region

329 has no homologous sequence in the genome of ZY05719. Thus, this trait in the cps locus of

330 NSUI002 strongly supports the hypothesis of capsular switch between SS2 and SS3, and favors a

331 possibility that the potential capsular switch between SS3 and SS2 might result from a

332 recombinational crossover point located ahead of cps3O. In summary, we find detailed evidence

333 for capsule switch within S.suis, at least and as expected, from the analysis of cps locus in CC28

334 SS2 and SS3 strains.

\section{Discussion}

336 Serotype 2 is the most prevalent serotype of $S$. suis worldwide. Among the major evolutionary

337 lineages revealed by genetics analyses, ST1 and 7 (CC1) SS2 are generally associated with 338 diseases (Goyette-Desjardins et al., 2014). However, ST25 (CC29) and ST28 (CC28), accounting

339 for larger proportions of SS2 strains in North American, present less virulence potentials in 340 animal model (Athey et al., 2015; Fittipaldi et al., 2011). In accordance, ST28 strains in China 341 are also regard as representive avirulent strains (Guo et al., 2020; Ma et al., 2020; Wang et al., 
2017). Those reports suggested that CC28 and CC29 SS2, which are phylogenetically distinct from CC1 SS2, are the pool of strains with lower virulence levels. In this study, we report that capsule switch exists in S. suis population, notably in CC28 and CC29 between SS2, SS3 and SS7. This finding may explain the genetic and phenotypic differences between CC1 SS2 and CC28/29 SS2, and indicate a possibility that CC28/29 SS2 was derived from an ancestor unrelated with CC1 SS2 through capsule switch, which has often been overshadowed by simple serotyping.

Capsule switch has been identified in other extracellular pathogens harboring polysaccharide capsule and natural competence, such as Neisseria meningitidis ( $N$, meningitidis), $S$. agalactiae and S. pneumoniae. Among which, the capsule switch of $S$. pneumoniae is the most representative and well-studied. Pneumococcal capsule switch can be achieved through gradual evolution with a combination of minor mutation, deletion and recombination in cps locus. For example, pneumococcal serotype 6A, 6B, 6C and 6D have near identity of cps locus, which only differ in wciP gene (Song et al., 2011). A similar case in S. suis is the capsule switch between SS1, SS2 and SS1/2 in CC1. Serotype 1/2 cannot be differentiated from serotype 1 and 2 by serum antigenicity (Perch et al., 1983), and genetic analysis of the cps locus demonstrated serotypes 1, 1/2 and 2 share the high genetic identities (Okura et al., 2013). On the other hand, capsule switch can occur through the exchange of large genomic fragment containing full cps locus (Bellais et al., 2012; Wyres et al., 2013), which is a suitable model to be applied in the capsule switch between SS 2, 3, 7 strains in CC28 and 29. Given the fact that cps locus is high diverse and variable between different $S$. suis serotypes (Okura et al., 2013), it is not logical to keep cps locus conserved or even identical when genetic backbone is subject to high degree of genetic rearrangement. Therefore, our data strongly supports a hypothesis that serotype 2, 3, 7 
capsule switch results from the exchange of large cps locus, leads to similar genetic backbones sharing common competence systems and defensive systems.

Importantly, the upstream and downstream flanking sequences of $c p s$ locus are almost identical between different $S$. suis serotypes (Okura et al., 2013), providing potential recombination sites for capsule switch. Furthermore, we studied the sequence of cps locus and flanking region between a CC1 SS2 ZY05719, CC28 SS2 strain NSUI002 and SS3 strain ST3 in detail. A genetic fragment was found to be conserved in the cps locus of CC28 SS3and SS2, but absent at that location in $\mathrm{CC} 1 \mathrm{SS}$, which suggests a potential recombination event occurred between CC28 SS2 and SS3. However, based on current information, we cannot determine the temporal relationship between strains, namely whether one is derived from another, or there is a common ancestor. In addition, although ST27 (a ST harboring both SS2 and SS3), ST28 (including NSUI002, SS2) and ST35 (including YB51 and ST3, SS3) are phylogenetically related and clustered together in CC28, ST35 SS2 or ST28 SS3 have not yet been observed, indicating that additional events occurred after capsule switch in this common evolutionary lineage. More collected isolates and sequenced genomes in the future would be helpful to address those issues.

In fact, the core of capsule switch is to increase diversity in the population and enhance fitness in certain environments, such as increasing antibiotic resistance or escaping herd immunity. Accumulated studies have demonstrated that capsule switch in S. pneumonia can be attributed to the selection pressure from the use of vaccines targeting capsule (Martins et al., 2010). Similarly, the change of CPS composition caused by cpsG mutation in $S$. iniae and capsule switch (from type III to IV) in CC17 S. agalactiae contributes to vaccine escape (Bellais et al., 2012; Heath et al., 2016). Besides that, capsule switch is also involved in bacterial pathogenicity. Pneumococcal capsule switch from $6 \mathrm{~A}$ to $6 \mathrm{C}$ promotes the resistance to complement system and presents 
enhanced virulence for respiratory tract infection (Sabharwal et al., 2014). Artificial capsule switch in S. pneumonia is able to alter virulence and infection outcomes in a mouse model (Kelly et al., 1994; Trzcinski et al., 2003). Although the role of capsule switch in S. suis is not clear, it is possible that the replacement of cps locus would cause phenotypic changes due to the alteration

392 of bacterial surface architecture. For instance, $N e u B$, an enzyme existing in serotype 1/2, 2 and 393 14, is essential for sialic acid biosynthesis (Feng et al., 2012). Therefore, acquiring NeuB may 394 increase bacterial resistance to complement system and phagocytosis (Feng et al., 2012; 395 Uchiyama et al., 2019). In fact, a recent study highlighted that experimentally switching capsule type 2 to 3 in S. suis leads to defective whole blood survival and bacterial virulence (Okura et al., 397 2020). More studies in the future are needed to clarify the phenotypic features of different $S$. suis 398 capsules, and what benefits bacteria may obtain from capsule switch.

\section{Acknowledgements}

This work was funded by the Natural Science Foundation of China (31972650), Shanghai 401 Agriculture Applied Technology Development Program (S0201700386), the Key Project of 402 Independent Innovation of the Fundamental Research Fund for the Central Universities of 403 Nanjing Agricultural University (KJQN201932) and the Priority Academic Program 404 Development of Jiangsu Higher Education Institutions (PAPD). We would like to thank Dr. 405 Qiang Li for his help in bioinformatic analyses.

\section{Conflict of interest}

407 The authors declare no conflicts of interest. 
The data that support the findings of this study are available from public database GENBANK, and the accession number is listed in Table. S2

\section{ORCID}

Huochun Yao https://orcid.org/0000-0002-3749-8557

\section{Ethical approval}

No animal experiments were performed thus ethical statement is not applicable in this study.

\section{References}

Athey, T.B., Auger, J.P., Teatero, S., Dumesnil, A., Takamatsu, D., Wasserscheid, J., Dewar, K., Gottschalk, M., Fittipaldi, N., 2015. Complex Population Structure and Virulence Differences among Serotype 2 Streptococcus suis Strains Belonging to Sequence Type 28. PLoS One 10, e0137760.

Bellais, S., Six, A., Fouet, A., Longo, M., Dmytruk, N., Glaser, P., Trieu-Cuot, P., Poyart, C., 2012. Capsular switching in group B Streptococcus CC17 hypervirulent clone: a future challenge for polysaccharide vaccine development. J Infect Dis 206, 1745-1752.

Darling, A.C., Mau, B., Blattner, F.R., Perna, N.T., 2004. Mauve: multiple alignment of conserved genomic sequence with rearrangements. Genome Res 14, 1394-1403.

Dong, W., Ma, J., Zhu, Y., Zhu, J., Yuan, L., Wang, Y., Xu, J., Pan, Z., Wu, Z., Zhang, W., Lu, C., Yao, H., 2015. Virulence genotyping and population analysis of Streptococcus suis serotype 2 isolates from China. Infect Genet Evol 36, 483-489.

Dong, W., Zhu, Y., Ma, Y., Ma, J., Zhang, Y., Yuan, L., Pan, Z., Wu, Z., Yao, H., 2017. Multilocus sequence typing and virulence genotyping of Streptococcus suis serotype 9 isolates revealed high genetic and virulence diversity. FEMS Microbiol Lett 364.

Dupuis, M.E., Villion, M., Magadan, A.H., Moineau, S., 2013. CRISPR-Cas and restrictionmodification systems are compatible and increase phage resistance. Nat Commun 4, 2087.

Feng, Y., Cao, M., Shi, J., Zhang, H., Hu, D., Zhu, J., Zhang, X., Geng, M., Zheng, F., Pan, X., Li, X., Hu, F., Tang, J., Wang, C., 2012. Attenuation of Streptococcus suis virulence by the alteration of bacterial surface architecture. Sci Rep 2, 710.

Fittipaldi, N., Segura, M., Grenier, D., Gottschalk, M., 2012. Virulence factors involved in the pathogenesis of the infection caused by the swine pathogen and zoonotic agent Streptococcus suis. Future Microbiol 7, 259-279.

Fittipaldi, N., Xu, J., Lacouture, S., Tharavichitkul, P., Osaki, M., Sekizaki, T., Takamatsu, D., Gottschalk, M., 2011. Lineage and virulence of Streptococcus suis serotype 2 isolates from North America. Emerg Infect Dis 17, 2239-2244.

Gerjets, I., Traulsen, I., Reiners, K., Kemper, N., 2011. Comparison of virulence gene profiles of Escherichia coli isolates from sows with coliform mastitis and healthy sows. Veterinary microbiology 152, 361-367. 
Goyette-Desjardins, G., Auger, J.P., Xu, J., Segura, M., Gottschalk, M., 2014. Streptococcus suis, an important pig pathogen and emerging zoonotic agent-an update on the worldwide distribution based on serotyping and sequence typing. Emerg Microbes Infect 3, e45.

Guo, G., Du, D., Yu, Y., Zhang, Y., Qian, Y., Zhang, W., 2020. Pan-genome analysis of Streptococcus suis serotype 2 revealed genomic diversity among strains of different virulence. Transbound Emerg Dis.

Heath, C., Gillen, C.M., Chrysanthopoulos, P., Walker, M.J., Barnes, A.C., 2016. Streptococcus iniae cpsG alters capsular carbohydrate composition and is a cause of serotype switching in vaccinated fish. Vet Microbiol 193, 116-124.

Higgins, R., Gottschalk, M., Boudreau, M., Lebrun, A., Henrichsen, J., 1995. Description of six new capsular types (29-34) of Streptococcus suis. J Vet Diagn Invest 7, 405-406.

Hill, J.E., Gottschalk, M., Brousseau, R., Harel, J., Hemmingsen, S.M., Goh, S.H., 2005. Biochemical analysis, cpn60 and 16S rDNA sequence data indicate that Streptococcus suis serotypes 32 and 34, isolated from pigs, are Streptococcus orisratti. Vet Microbiol 107, 63-69.

Kelly, T., Dillard, J.P., Yother, J., 1994. Effect of genetic switching of capsular type on virulence of Streptococcus pneumoniae. Infect Immun 62, 1813-1819.

Kerdsin, A., Akeda, Y., Hatrongjit, R., Detchawna, U., Sekizaki, T., Hamada, S., Gottschalk, M., Oishi, K., 2014. Streptococcus suis serotyping by a new multiplex PCR. J Med Microbiol 63, 824-830.

Kerdsin, A., Hatrongjit, R., Gottschalk, M., Takeuchi, D., Hamada, S., Akeda, Y., Oishi, K., 2017. Emergence of Streptococcus suis serotype 9 infection in humans. J Microbiol Immunol Infect 50, 545-546.

King, S.J., Leigh, J.A., Heath, P.J., Luque, I., Tarradas, C., Dowson, C.G., Whatmore, A.M., 2002. Development of a multilocus sequence typing scheme for the pig pathogen Streptococcus suis: identification of virulent clones and potential capsular serotype exchange. J Clin Microbiol 40, 3671-3680.

Kobayashi, N., Lee, K., Yamazaki, A., Saito, S., Furukawa, I., Kono, T., Maeda, E., Isobe, J., Sugita-Konishi, Y., Hara-Kudo, Y., 2013. Virulence gene profiles and population genetic analysis for exploration of pathogenic serogroups of Shiga toxin-producing Escherichia coli. J Clin Microbiol 51, 4022-4028.

Ma, J., Zhang, Z., Pan, Z., Bai, Q., Zhong, X., Zhu, Y., Zhang, Y., Wu, Z., Liu, G., Yao, H., 2020. Streptococcus suis Uptakes Carbohydrate Source from Host Glycoproteins by Nglycans Degradation System for Optimal Survival and Full Virulence during Infection. Pathogens 9.

Martins, E.R., Melo-Cristino, J., Ramirez, M., 2010. Evidence for rare capsular switching in Streptococcus agalactiae. J Bacteriol 192, 1361-1369.

Mateus, L., Henriques, S., Merino, C., Pomba, C., Lopes da Costa, L., Silva, E., 2013. Virulence genotypes of Escherichia coli canine isolates from pyometra, cystitis and fecal origin. Vet Microbiol 166, 590-594.

Okura, M., Auger, J.-P., Shibahara, T., Goyette-Desjardins, G., Calsteren, M.-R.V., Maruyama, F., Kawai, M., Osaki, M., Segura, M., Gottschalk, M., Takamatsu, D., 2020. Capsular polysaccharide switching in Streptococcus suis modulates host cell interactions and virulence. bioRxiv, 2020.2010.2012.336958.

Okura, M., Nozawa, T., Watanabe, T., Murase, K., Nakagawa, I., Takamatsu, D., Osaki, M., Sekizaki, T., Gottschalk, M., Hamada, S., Maruyama, F., 2017. A Locus Encoding 
Variable Defense Systems against Invading DNA Identified in Streptococcus suis. Genome Biol Evol 9, 1000-1012.

Okura, M., Takamatsu, D., Maruyama, F., Nozawa, T., Nakagawa, I., Osaki, M., Sekizaki, T., Gottschalk, M., Kumagai, Y., Hamada, S., 2013. Genetic analysis of capsular polysaccharide synthesis gene clusters from all serotypes of Streptococcus suis: potential mechanisms for generation of capsular variation. Appl Environ Microbiol 79, 2796-2806.

Okwumabua, O., O'Connor, M., Shull, E., 2003. A polymerase chain reaction (PCR) assay specific for Streptococcus suis based on the gene encoding the glutamate dehydrogenase. FEMS Microbiol Lett 218, 79-84.

Pan, Z., Ma, J., Dong, W., Song, W., Wang, K., Lu, C., Yao, H., 2015. Novel variant serotype of streptococcus suis isolated from piglets with meningitis. Appl Environ Microbiol 81, 976-985.

Perch, B., Pedersen, K.B., Henrichsen, J., 1983. Serology of capsulated streptococci pathogenic for pigs: six new serotypes of Streptococcus suis. J Clin Microbiol 17, 993-996.

Qiu, X., Bai, X., Lan, R., Zheng, H., Xu, J., 2016. Novel Capsular Polysaccharide Loci and New Diagnostic Tools for High-Throughput Capsular Gene Typing in Streptococcus suis. Appl Environ Microbiol 82, 7102-7112.

Rasmussen, G., Monecke, S., Ehricht, R., Soderquist, B., 2013. Prevalence of clonal complexes and virulence genes among commensal and invasive Staphylococcus aureus isolates in Sweden. PloS one 8, e77477.

Richter, M., Rossello-Mora, R., 2009. Shifting the genomic gold standard for the prokaryotic species definition. Proc Natl Acad Sci U S A 106, 19126-19131.

Sabharwal, V., Stevenson, A., Figueira, M., Orthopoulos, G., Trzcinski, K., Pelton, S.I., 2014. Capsular switching as a strategy to increase pneumococcal virulence in experimental otitis media model. Microbes Infect 16, 292-299.

Song, J.H., Baek, J.Y., Ko, K.S., 2011. Comparison of capsular genes of Streptococcus pneumoniae serotype 6A, 6B, 6C, and 6D isolates. J Clin Microbiol 49, 1758-1764.

Tien, L.H.T., Nishibori, T., Nishitani, Y., Nomoto, R., Osawa, R., 2013. Reappraisal of the taxonomy of Streptococcus suis serotypes 20, 22, 26, and 33 based on DNA-DNA homology and sodA and recN phylogenies. Vet Microbiol 162, 842-849.

Trzcinski, K., Thompson, C.M., Lipsitch, M., 2003. Construction of otherwise isogenic serotype 6B, 7F, 14, and 19F capsular variants of Streptococcus pneumoniae strain TIGR4. Appl Environ Microbiol 69, 7364-7370.

Uchiyama, S., Sun, J., Fukahori, K., Ando, N., Wu, M., Schwarz, F., Siddiqui, S.S., Varki, A., Marth, J.D., Nizet, V., 2019. Dual actions of group B Streptococcus capsular sialic acid provide resistance to platelet-mediated antimicrobial killing. Proc Natl Acad Sci U S A 116, 7465-7470.

Wang, J., Feng, Y., Wang, C., Zheng, F., Hassan, B., Zhi, L., Li, W., Yao, Y., He, E., Jiang, S., 2017. Genome-wide analysis of a avirulent and reveal the strain induces pro-tective immunity against challenge with virulent Streptococcus suis Serotype 2. BMC microbiology 17, 67.

Wei, Z., Li, R., Zhang, A., He, H., Hua, Y., Xia, J., Cai, X., Chen, H., Jin, M., 2009. Characterization of Streptococcus suis isolates from the diseased pigs in China between 2003 and 2007. Vet Microbiol 137, 196-201.

Wiedenheft, B., Sternberg, S.H., Doudna, J.A., 2012. RNA-guided genetic silencing systems in bacteria and archaea. Nature 482, 331-338. 
Willemse, N., Schultsz, C., 2016. Distribution of Type I Restriction-Modification Systems in Streptococcus suis: An Outlook. Pathogens 5.

Wyres, K.L., Lambertsen, L.M., Croucher, N.J., McGee, L., von Gottberg, A., Linares, J., Jacobs, M.R., Kristinsson, K.G., Beall, B.W., Klugman, K.P., Parkhill, J., Hakenbeck, R., Bentley, S.D., Brueggemann, A.B., 2013. Pneumococcal capsular switching: a historical perspective. J Infect Dis 207, 439-449.

Yao, X., Li, M., Wang, J., Wang, C., Hu, D., Zheng, F., Pan, X., Tan, Y., Zhao, Y., Hu, L., Tang, J., Hu, F., 2015. Isolation and characterization of a native avirulent strain of Streptococcus suis serotype 2: a perspective for vaccine development. Sci Rep 5, 9835.

Yother, J., 2011. Capsules of Streptococcus pneumoniae and other bacteria: paradigms for polysaccharide biosynthesis and regulation. Annu Rev Microbiol 65, 563-581.

Zhu, W., Wu, C., Sun, X., Zhang, A., Zhu, J., Hua, Y., Chen, H., Jin, M., 2013. Characterization of Streptococcus suis serotype 2 isolates from China. Vet Microbiol 166, 527-534.

Zhu, Y., Dong, W., Ma, J., Yuan, L., Hejair, H.M., Pan, Z., Liu, G., Yao, H., 2017. Characterization and virulence clustering analysis of extraintestinal pathogenic Escherichia coli isolated from swine in China. BMC Vet Res 13, 94.

Zhu, Y., Dong, W., Ma, J., Zhang, Y., Pan, Z., Yao, H., 2019. Utilization of the ComRS system for the rapid markerless deletion of chromosomal genes in Streptococcus suis. Future Microbiol 14, 207-222.

\section{Figure legends}

Figure 1. Clustering of SS2, SS3 and SS7 isolates based on the profiles of virulence related

genes. The lateral axis of the matrix is the set of 19 genes and the vertical axis is the set of 88 strains. Green color covers SS2 strains, yellow covers SS3 strains and pink covers SS7 strains. Each black square refers to a positive detection of a specific virulence related gene in a single $S$. suis strain. Agglomerative clustering was performed using the unweighted average linkage (UPGMA) with the BioNumerics software.

Figure 2. eBURST analysis of $S$. suis MLST data. (A) eBURST software was used to analyze the MLST data of whole S. suis population. A total of 9 major CCs were identified, including CC1, CC16, CC28, CC29, CC94, CC104, CC201, CC423 and CC528. (B) Detailed analysis all SS2, SS3 and SS7 strains. SS2 strains are colored in back, SS3 in green and SS7 in purple. ST1 
and ST7 SS2 are clustered into CC1. CC28 includes both SS2 and SS3 strains, whereas CC29 contains SS2 and SS7 strains.

Figure 3. Phylogenetic tree of 25 S. suis strains based on orthologous gene clusters. The phylogenetic tree was constructed using neighbor-joining (NJ) method. S. suis strains of different serotypes can be classified into 3 major clades. Clade 1 appeared to be phylogenetic distinct from Clade 2. Clade 1 harbors virulent SS2 strains, and Clade 2 SS3 strains, SS7 strain and SS2 strains with lower virulence level.

\section{Figure 4. Multigenome comparison between Clade 1 and Clade 2 S. suis strains obtained by}

Mauve tool. Each colored region refers to a locally collinear block (LCB). Colors are arbitrarily assigned by software to each LCB. The vertical peaks in each LCB denotes the variance of conservation. The LCBs below the center line of genomes are in reverse complement orientation. As reference genome, Clade 2 SS2 strain NSUI002 are compared with SS3 strains (ST3 and YB51) and Clade 1 SS2 strain ZY05719. The Multigenome comparison of SS2 strain ZY05719, NSUI060 and SS7 strain D9 is shown in Figure S1.

Figure 5. Heat map based on ANI values between every two genome sequences. The average nucleotide identity (ANI) were calculated using the web-based EZ BioCloud platform. Heatmaps based on ANI values were generated with HemI 1.0 (Heatmap Illustrator software, version 1.0).

Figure 6. The structures of defense systems in S. suis isolates. (A) Three types of type I RM systems are found in S. suis. Type A and B RM systems only present in Clade $1 S$. suis strains, and Type $\mathrm{C}$ was common RM system appearing in all of $S$. suis strains (Both Clade 1 and Clade 2). (B) Sequences of CRISPR repeats in representative S. suis strains. (C) The structures of competence and CRISPR elements in S. suis. Only Clade 2 strains harbors CRISPRs. Clade 1 
591 and Clade 2 strains have different sequences in ComRS competence systems. The ComX

592 regulator are conserved in S. sui strains, but Clade 2 strains have one more copy of ComX.

593 Figure 7. S. suis transformation induced by two different XIPs. Peptide XIP induces $S$. suis

594 competence to uptake exogenous DNA. The competence effieiency can be assessed by positive

595 transformants grow on THB $(s p c: c m+)$. Type A XIP is only able to induce transformation in

596 Clade 1 SS2 strains. Type B XIP induces transformation in SS2, SS3, SS7 strains belonging to

597 Clade 2.

598 Figure 8. Schematic representations of cps locus in SS2 and SS3 strains. The cps locus of 599 Clade 2 SS2 strain NSUI002, Clade 2 SS3 strain ST3 and Clade 1 SS2 strain ZY05719 were 600 compared. The flanking regions of cps locus reveals high similarity. Red arrow indicates a 601 translocation event. Red line box shows a region uniquely present in the cps locus of both ST3 602 and NSUI002.

604 Table 1 Existing capsule switch in S. suis

\begin{tabular}{cc}
\hline S. suis sequence type (ST) & S. suis serotypes \\
\hline ST1 & SS1/2, SS1, SS2, SS8, SS9, SS14 \\
ST13 & SS1, SS14 \\
ST15 & SS3, SS9 \\
ST16 & SS4, SS9 \\
ST17 & SS4, SS5 \\
ST27 & SS2, SS3 \\
ST28 & SS1/2, SS2 \\
ST29 & SS2, SS3, SS7 \\
ST65 & SS15, SS27
\end{tabular}


bioRxiv preprint doi: $h t t p s: / / d o i . o r g / 10.1101 / 2020.11 .11 .377622$; this version posted November 11,2020 . The copyright holder for this preprint (which was not certified by peer review) is the author/funder, who has granted bioRxiv a license to display the preprint in perpetuity. It is made available under aCC-BY-NC-ND 4.0 International license.

ST76

ST89

ST94

ST105

ST136

ST156

ST243

ST297
SS17, SS19

SS3, SS7

SS4, SS16

SS2, SS14

SS7, SS9

SS1, SS2

SS2, SS9

SS7, SS9

605 


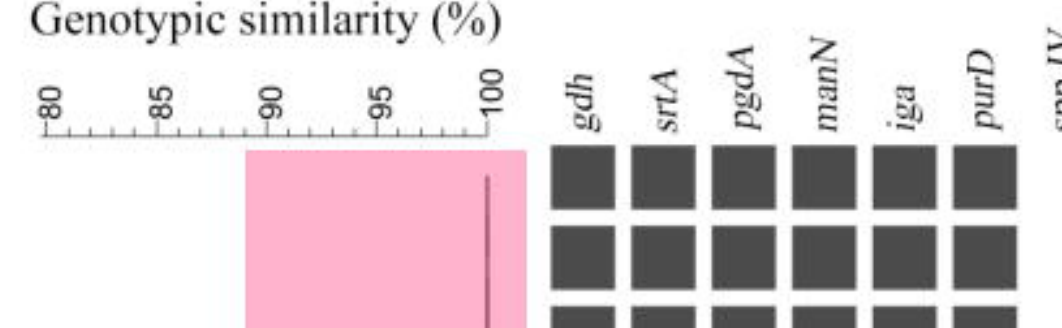

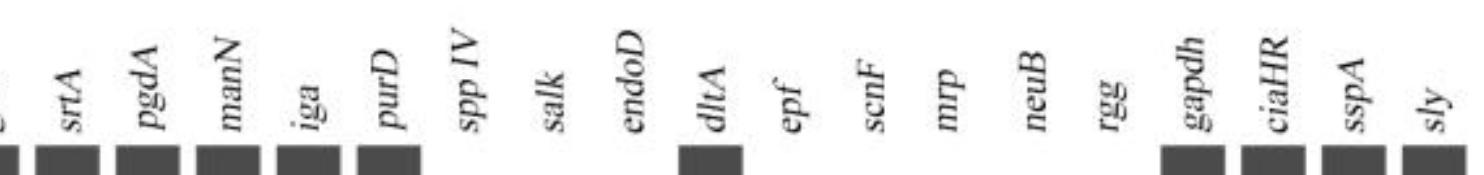

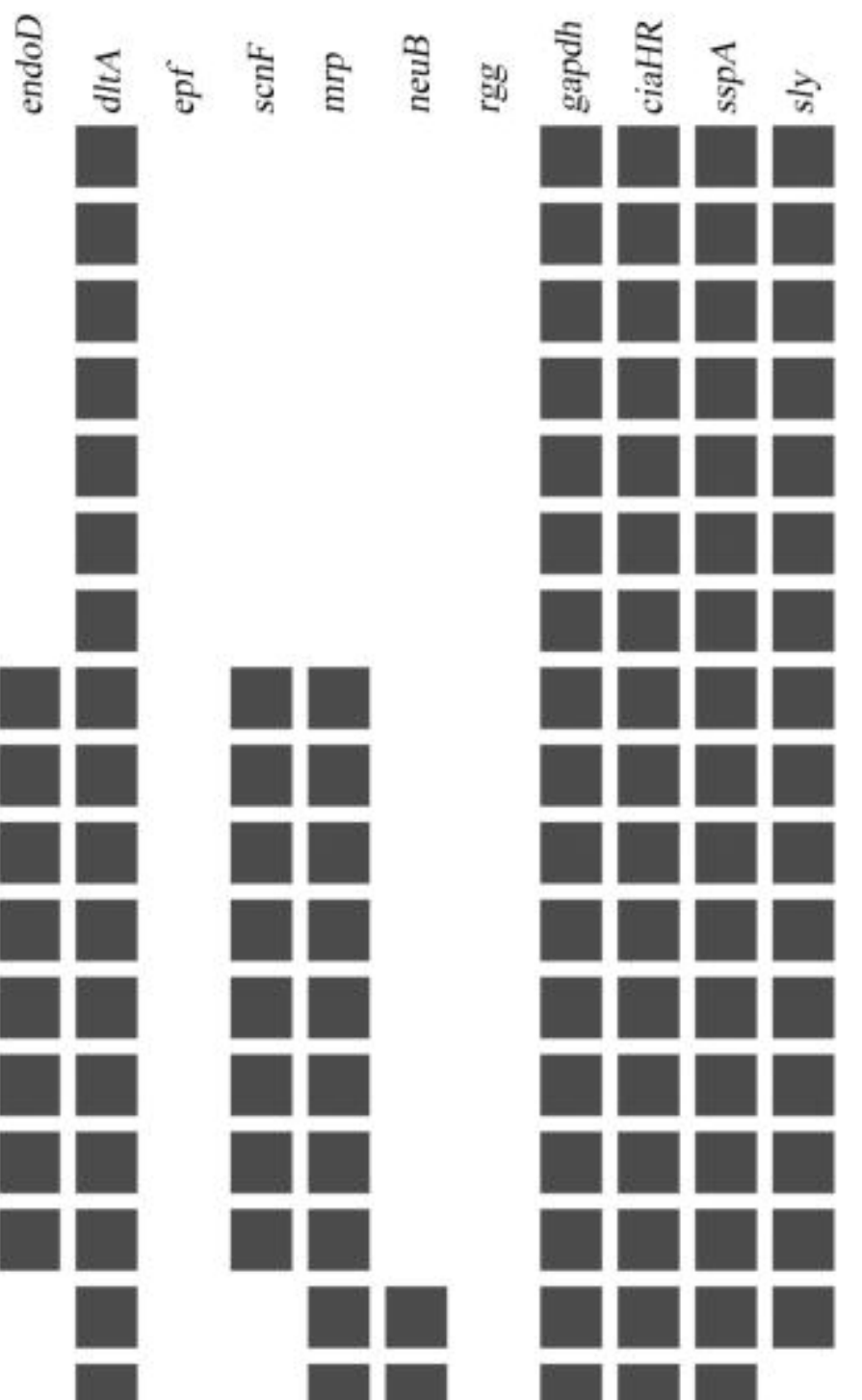
+口qu 4982 9日2 tan

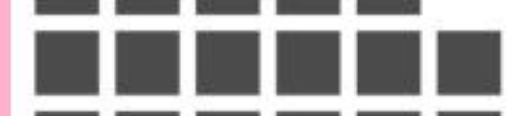
98010

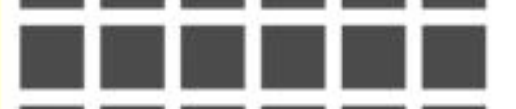

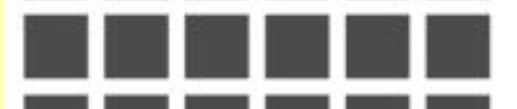
1970 40 早早

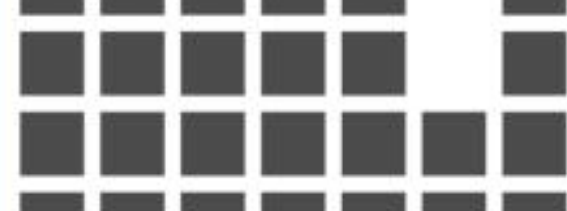

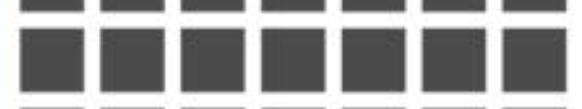

Cluster I PQpan PQtan

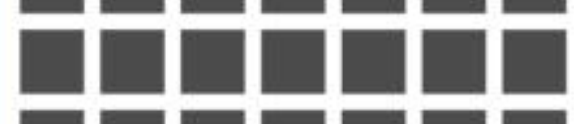
प日ung 且且 1989

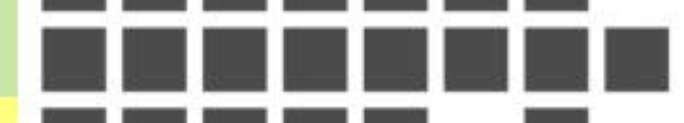
口qu 口n

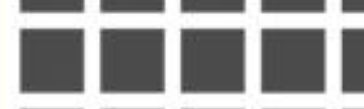
口qu 口n 口且 $\square \square \square$ G g日E

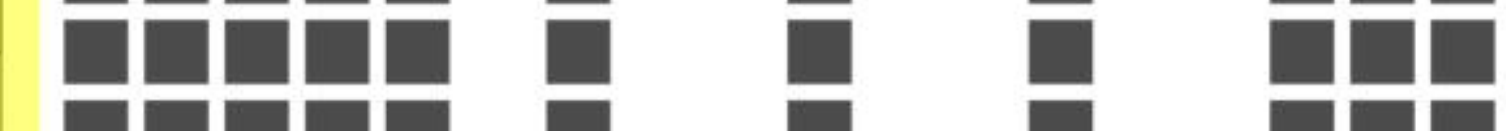
口q⿴囗十 口qu日e Prana

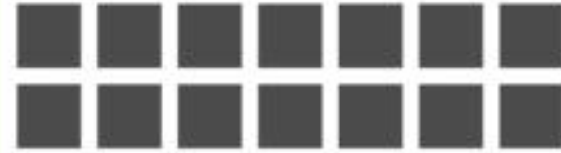
appan QPि⿴囗十 tgta 口q⿴囗十 Pq⿴囗十 pq⿴囗十 anta 口n⿴囗十 口回且

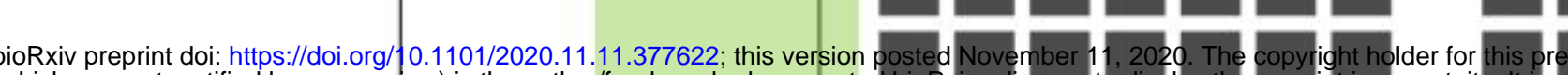
onger पम中मnमि ananana

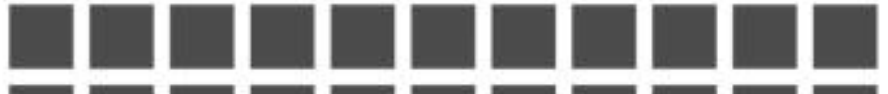

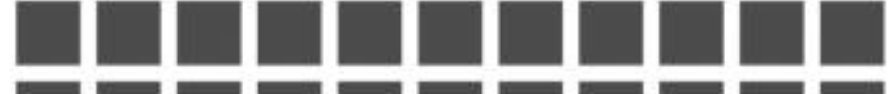

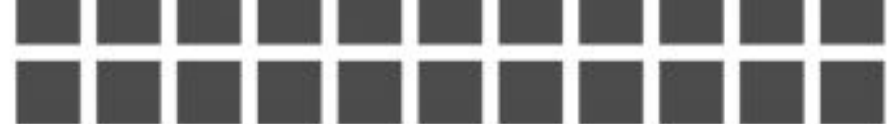
tetptgt gragng Qquangan rifiriting -qungu

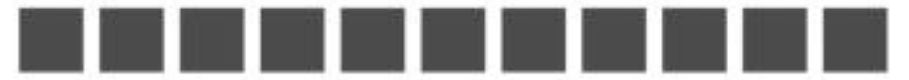

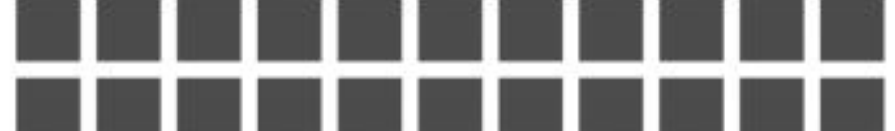

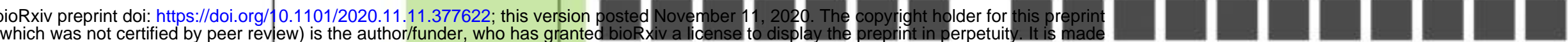

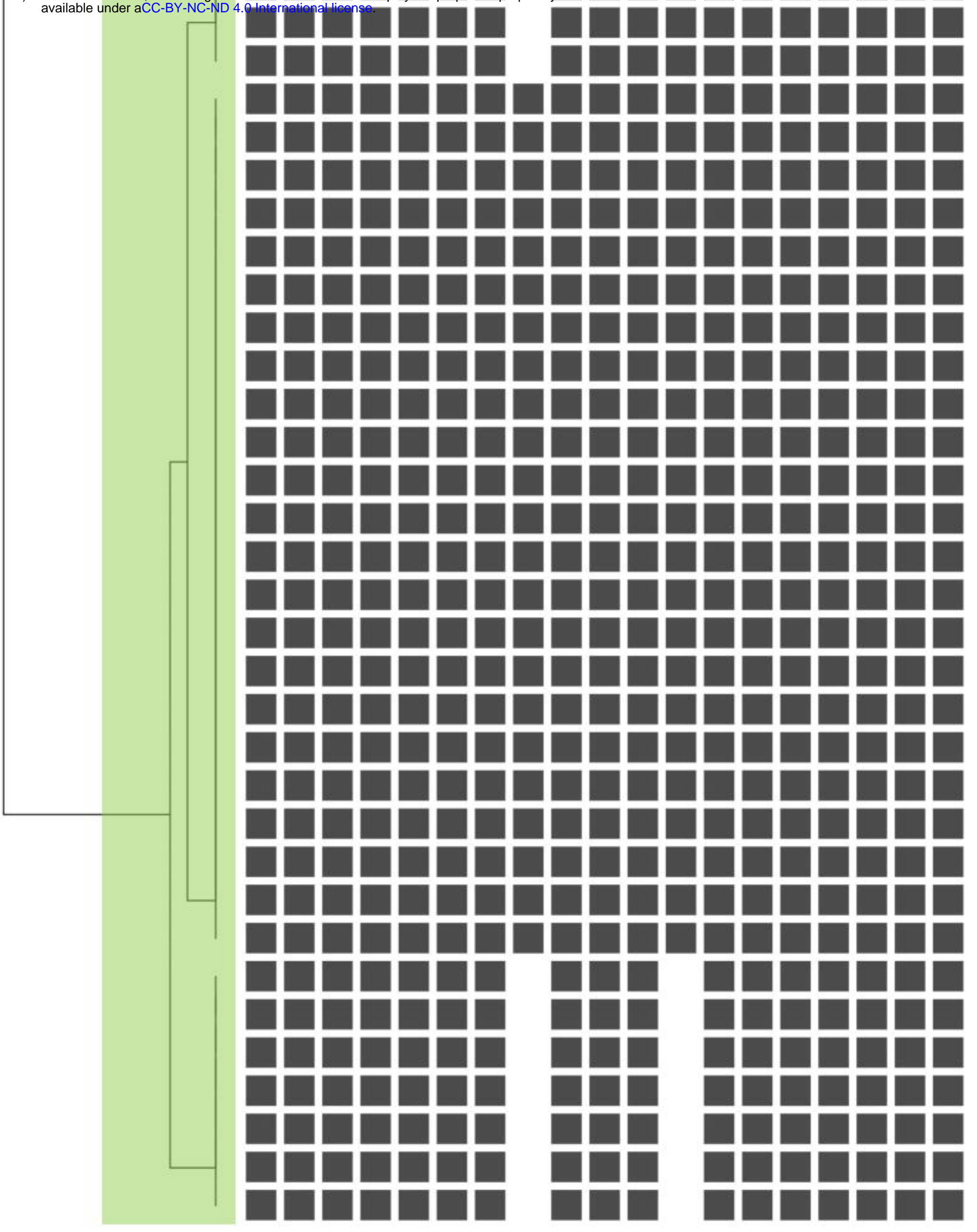




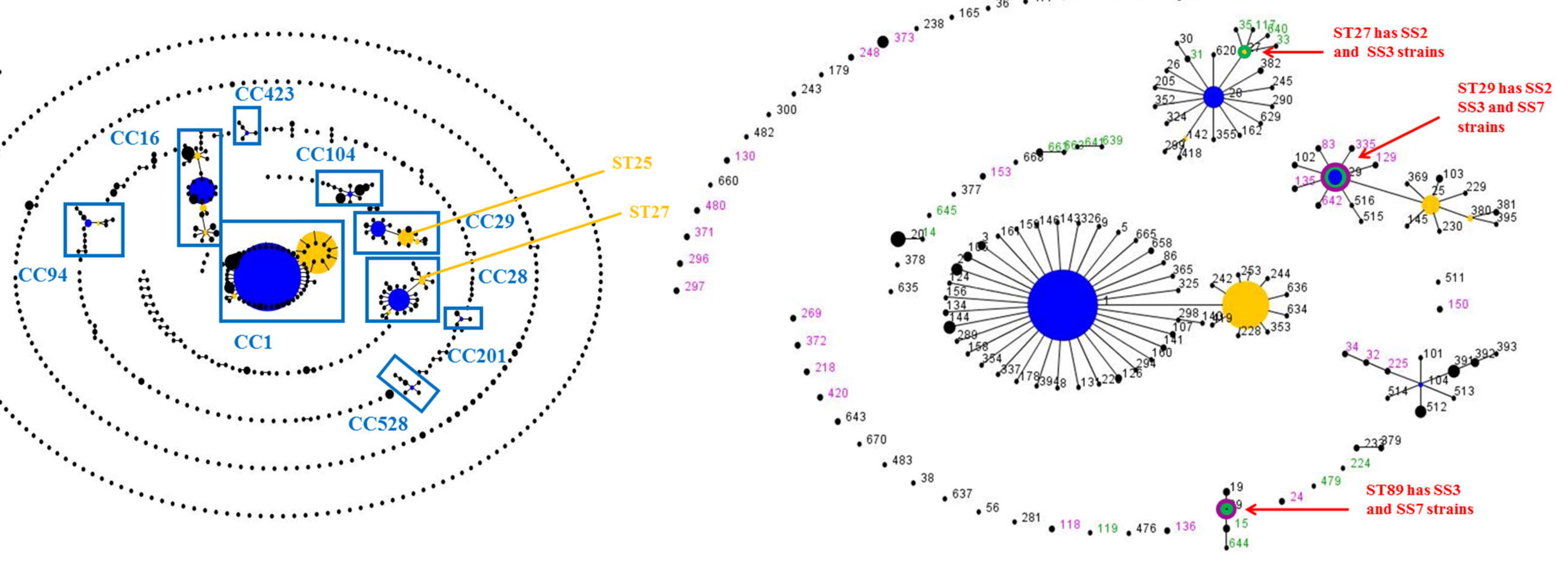




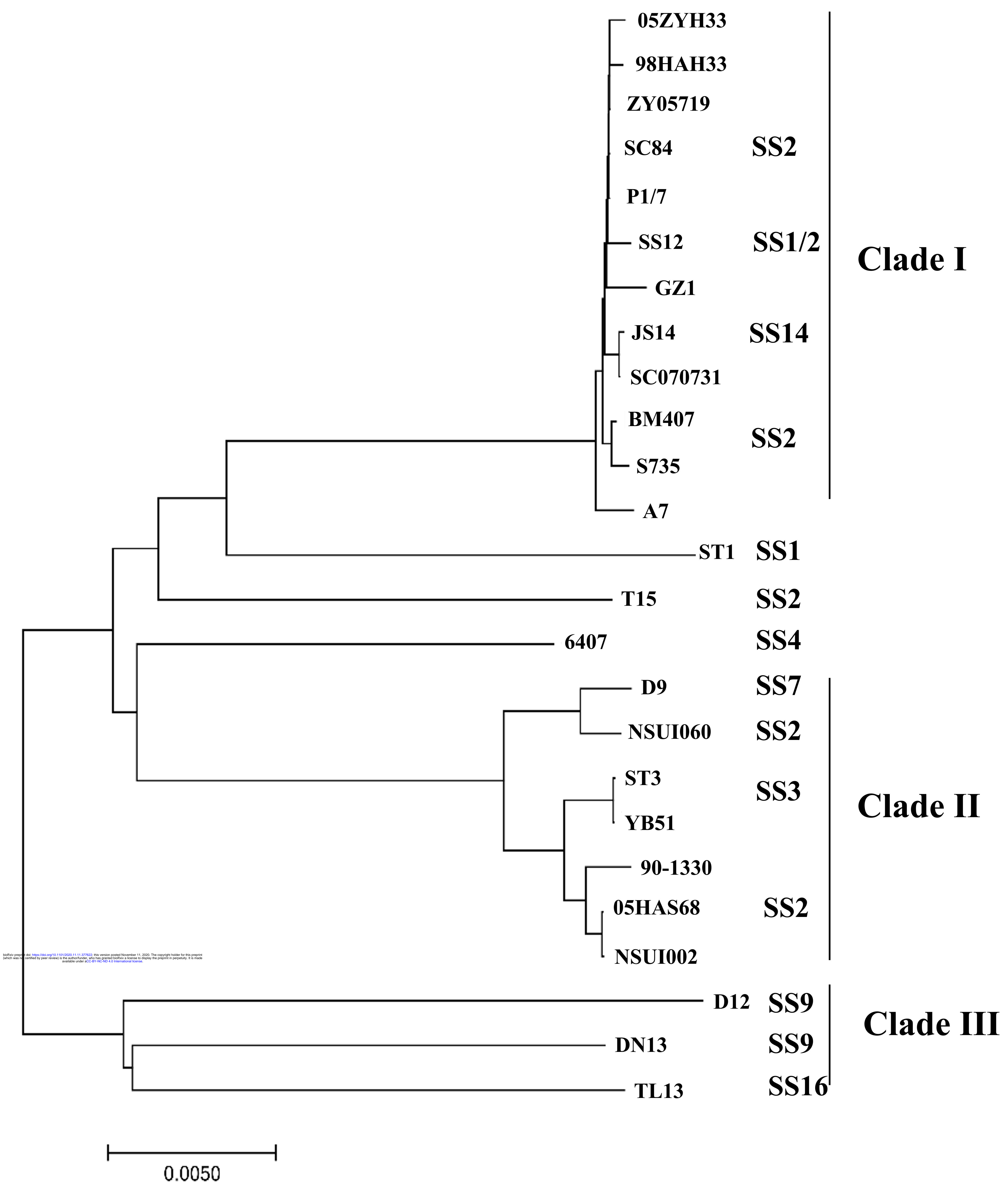




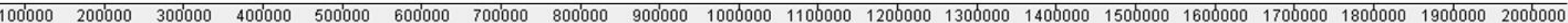

SS3 YB51

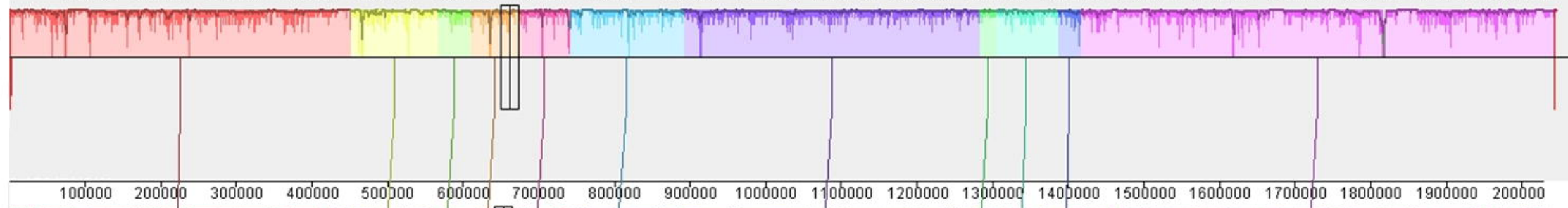

SS3 ST3

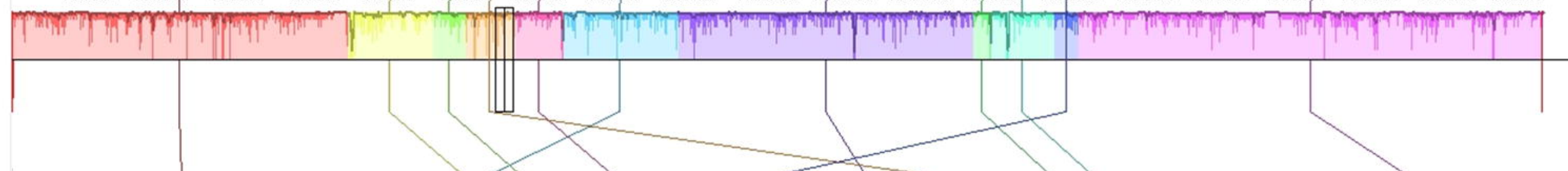

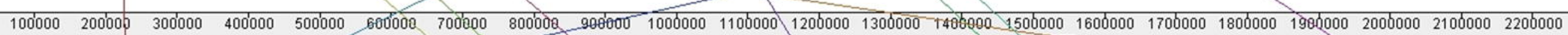

SS2 NSUI002
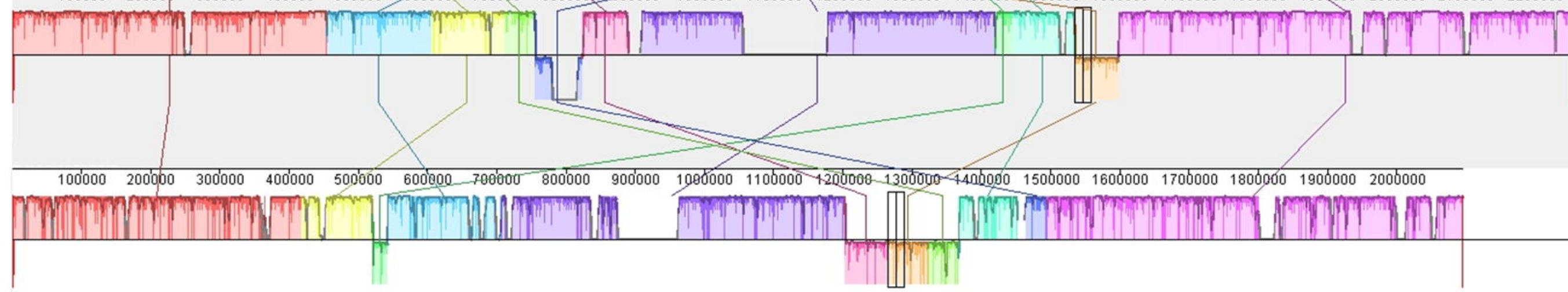


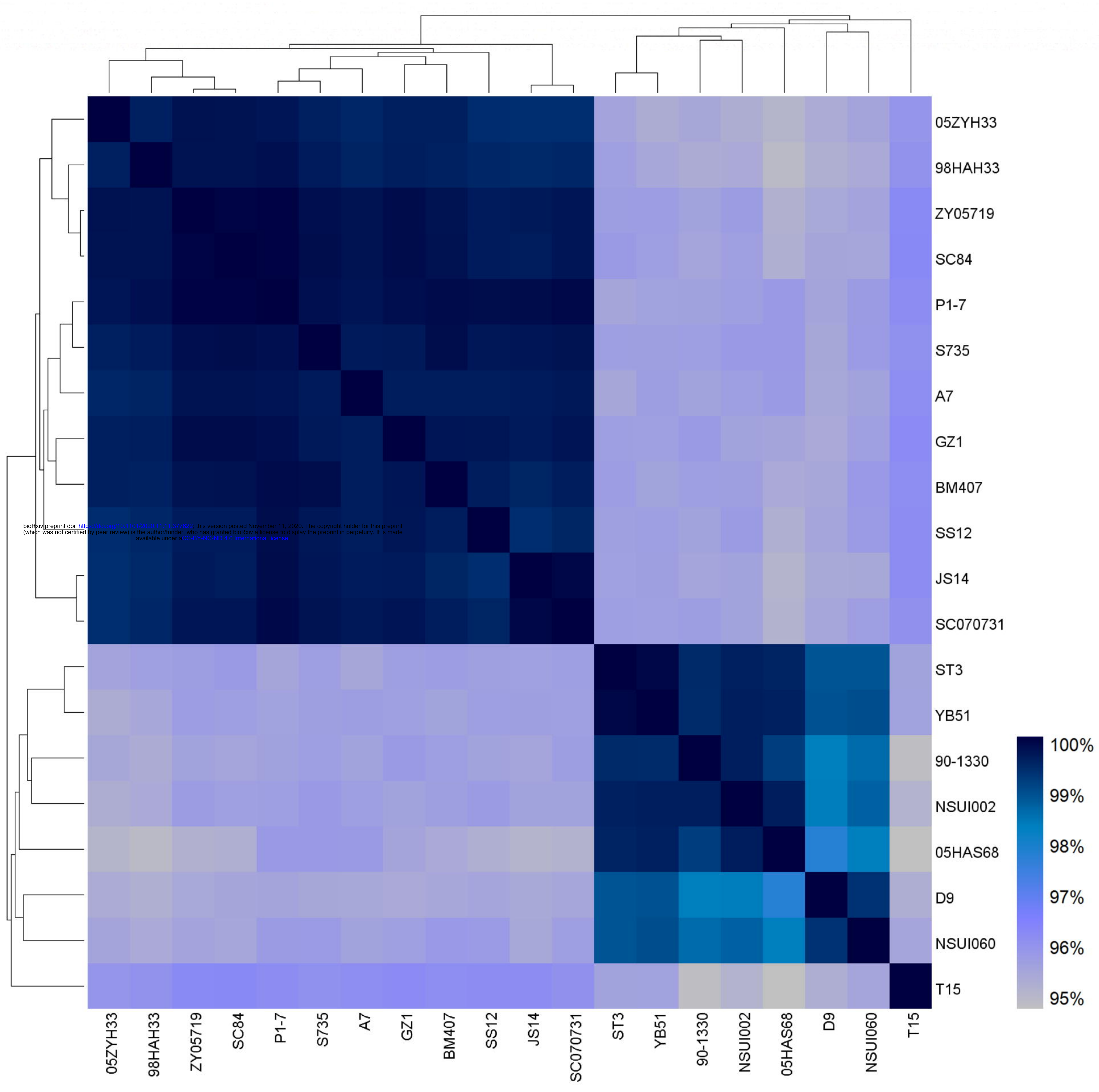


A

Type A

Type B

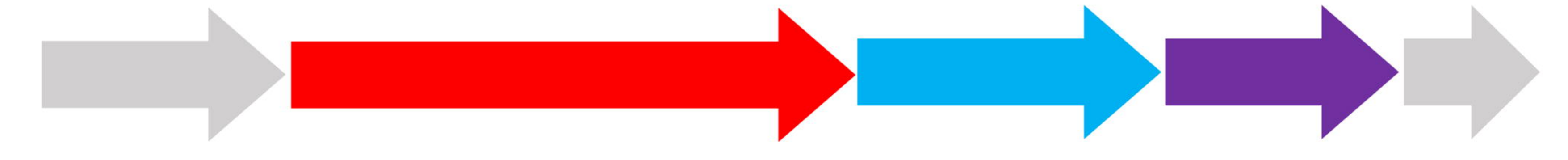

$h s d S 1$

B

$h s d \mathrm{M}$

“core" RM type I system

Type C

$\mathrm{C}$

\begin{tabular}{ll} 
& ST \\
ZY05719 & 7 \\
P177 & \\
\hline
\end{tabular}

NSUI060

25

NSUI002

28

ST3

35

D9

29

Competence genes

ComR ComS ComX1 ComX2
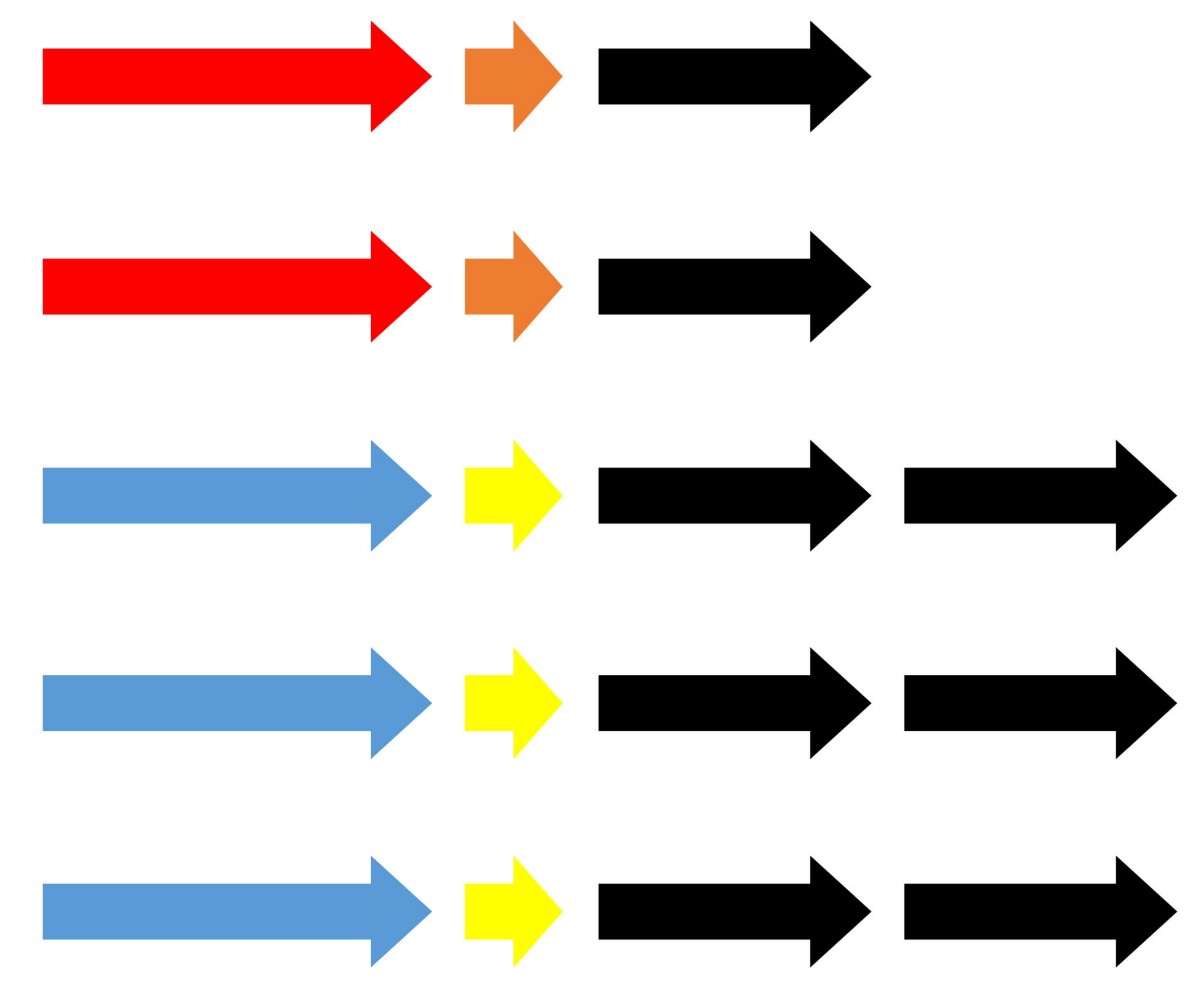

Type I RM system

type A type B type C

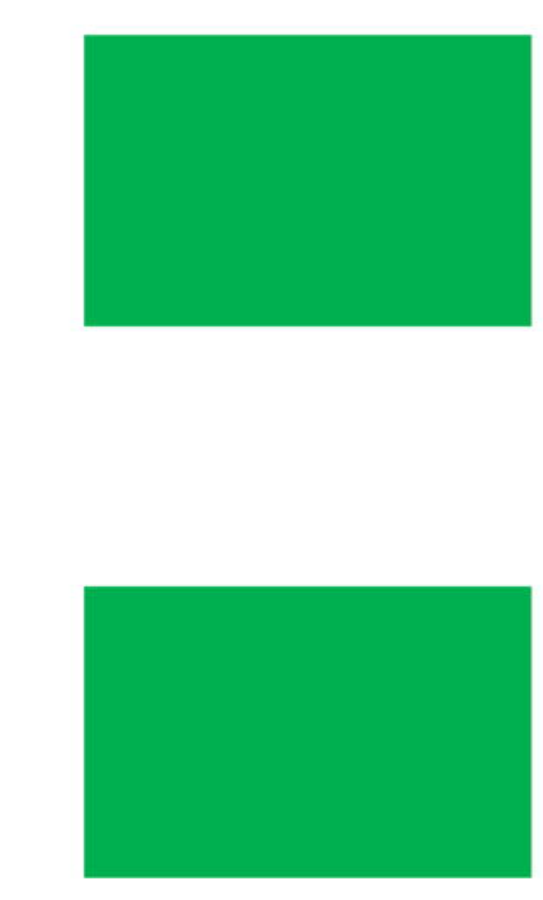

\section{CRISPR}

CRISPR CRISPR*
Consensus GTTTTACTGT TACTTAAATCTTGAGAGTACAAAAACXXX Length

NSUI002 GTTTTACTGTTACTTAAATCTTGAGAGTACAAAAAC NSUI060 GTTTTACTGTTACTTAAATCTTGAGAGTACAAAAAC

ST3 GTTTTACTGTTACTTAAATCTTGAGAGTACAAAAAC

D9-1 GTTTTACTGTTACTTAAATCTTGAGAGTACAAAAAC

D9-2 GTTTTACTGTTACTTAAATCTTGAGAGTACAAAAACTTA 

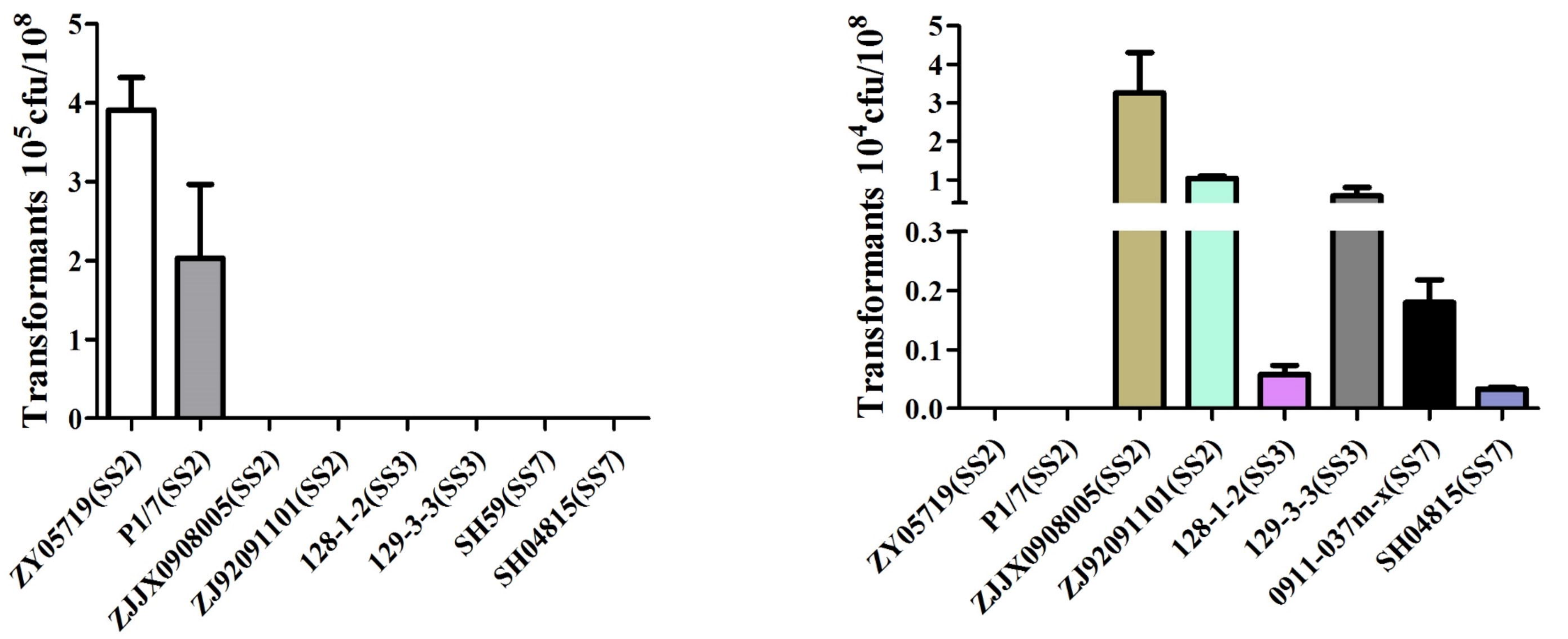

Type A XIP

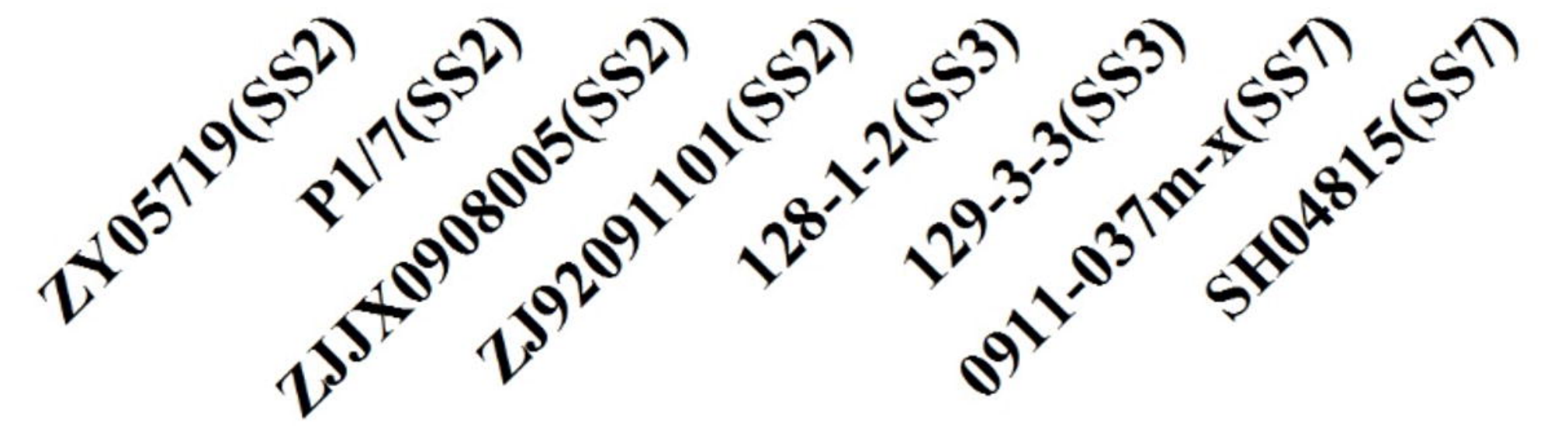

Type B XIP 


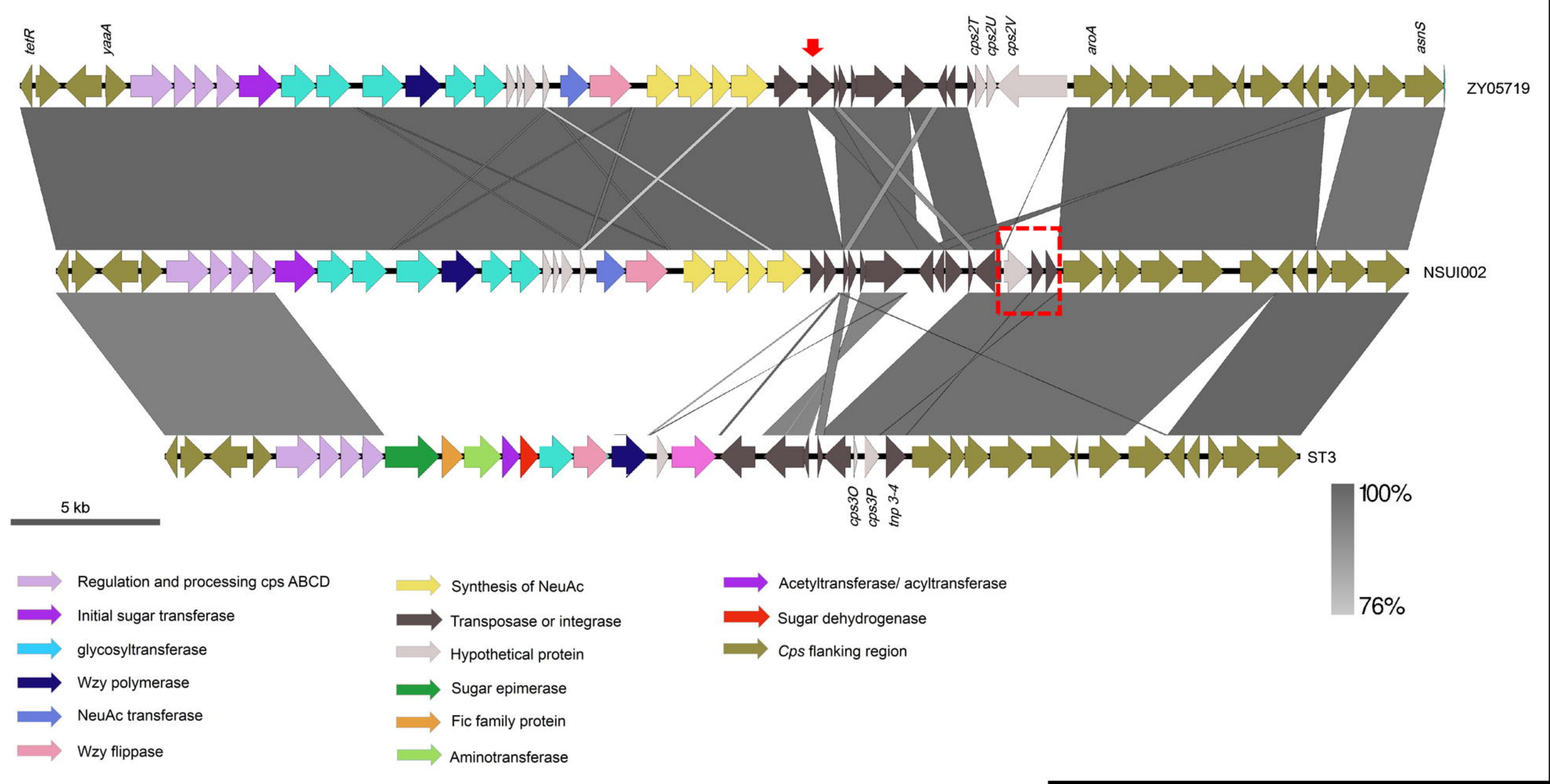

